\title{
In situ imaging of the conducting filament in a silicon oxide resistive switch
}

SUBJECT AREAS:

ELECTRONIC MATERIALS

AND DEVICES

ELECTROCHEMISTRY

MATERIALS SCIENCE

IMAGING

Received

30 September 2011

Accepted

19 December 2011

Published

31 January 2012

Correspondence and requests for materials should be addressed to J.M.T. (tour@rice.edu)
Jun Yao', Lin Zhong ${ }^{2,3}$, Douglas Natelson ${ }^{2,4}$ \& James M. Tour ${ }^{3,5}$

\begin{abstract}
'Applied Physics Program through the Department of Bioengineering, Rice University, 6100 Main St., Houston, Texas 77005, ${ }^{2}$ Department of Electrical and Computer Engineering, Rice University, 6100 Main St., Houston, Texas 77005, ${ }^{3}$ Department of Computer Science, Rice University, 6100 Main St., Houston, Texas 77005, ${ }^{4}$ Department of Physics and Astronomy, Rice University, 6100 Main St., Houston, Texas 77005, ${ }^{5}$ Departments of Chemistry and Mechanical Engineering and Materials Science, Rice University, 6100 Main St., Houston, Texas 77005.
\end{abstract}

The nature of the conducting filaments in many resistive switching systems has been elusive. Through in situ transmission electron microscopy, we image the real-time formation and evolution of the filament in a silicon oxide resistive switch. The electroforming process is revealed to involve the local enrichment of silicon from the silicon oxide matrix. Semi-metallic silicon nanocrystals with structural variations from the conventional diamond cubic form of silicon are observed, which likely accounts for the conduction in the filament. The growth and shrinkage of the silicon nanocrystals in response to different electrical stimuli show energetically viable transition processes in the silicon forms, offering evidence for the switching mechanism. The study here also provides insights into the electrical breakdown process in silicon oxide layers, which are ubiquitous in a host of electronic devices.

nderstanding the mechanisms in resistive switching materials is essential for applications in future nonvolatile memory and logic devices ${ }^{1-3}$. When in the $\mathrm{ON}$ state, conduction in such systems can occur through a filament rather than the bulk channel. While filament confinement is considered advantageous $^{4}$, it presents challenges in mechanistic probing. Surface conductance mapping provides useful information ${ }^{5-7}$, yet the embedded nature of the filament makes further data difficult to obtain. Transmission electron microscopy (TEM) revealed structural and compositional details in the filaments ${ }^{8-10}$. However, the isolation of the filament involved in this ex situ imaging ${ }^{8-10}$ is destructive and potentially alters it. Here we demonstrate the in situ imaging of the $\mathrm{Si}$ filament in a silicon oxide $\left(\mathrm{SiO}_{\mathrm{x}}, x \sim 2\right)$ resistive switch. The $\mathrm{Si}$ nanocrystals are observed to be semimetallic. The growth and shrinkage of the silicon nanocrystals offer insights into the switching mechanism. This could also have implications in a wide range of silicon oxide breakdown phenomena.

$\mathrm{SiO}_{\mathrm{x}}$ resistive switching memory is attractive for its fully CMOS (complementary metal-oxide-semiconductor) compatible material composition and processing ${ }^{4}$. Two types of resistive switching memory have been constructed based on $\mathrm{SiO}_{\mathrm{x}}$. The first type belongs to the programmable metallization cell ${ }^{4}$, which relies on the formation and rupture of the metal filament injected from the electrode ${ }^{11}$. It features bipolar switching behavior and is extrinsic to $\mathrm{SiO}_{\mathrm{x}}$ with $\mathrm{SiO}_{\mathrm{x}}$ merely serving as the passive solid electrolyte. The second type shows unipolar current-voltage $(I-V)$ characteristics with the reset voltage larger than the set value ${ }^{9}$ (Fig. 1a). The filamentary switching was recently revealed to be the intrinsic property of $\mathrm{SiO}_{\mathrm{x}}$ as it is electrode-independent ${ }^{9,12}$. The study here focuses on this type of intrinsic resistive switching in $\mathrm{SiO}_{\mathrm{x}}$. While ex situ imaging revealed a silicon-rich switching site, information regarding conduction and switching mechanisms was largely missing.

Through in situ TEM, we demonstrate the real-time imaging of the formation and evolution of the filament in a silicon oxide resistive switch. The electroforming process is shown to involve the local enrichment of silicon from the silicon oxide matrix. Semi-metallic silicon nanocrystals with structural variations from the conventional diamond cubic form of silicon are observed, which likely accounts for the conduction in the filament. The growth and shrinkage of the silicon nanocrystals in response to different electrical stimuli show energetically viable transition processes in the silicon forms, offering evidence for the switching mechanism. Since soft breakdown is involved in the electroforming process ${ }^{1}$, the study here could also provide insights into the general electrical breakdown process in $\mathrm{SiO}_{\mathrm{x}}$, a material that plays a ubiquitous role in semiconductor electronics.

\section{Results}

Figs. $1 b-d$ shows the schematics of the device design and setup for the in situ imaging. The imaging region consists of $\mathrm{a} \mathrm{SiO}_{\mathrm{x}}$ thin-walled structure covered by a layer of amorphous carbon $(\alpha-\mathrm{C})$, which is connected to 
a

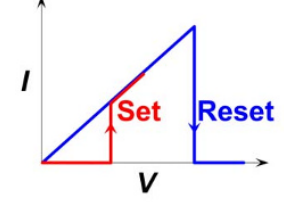

b
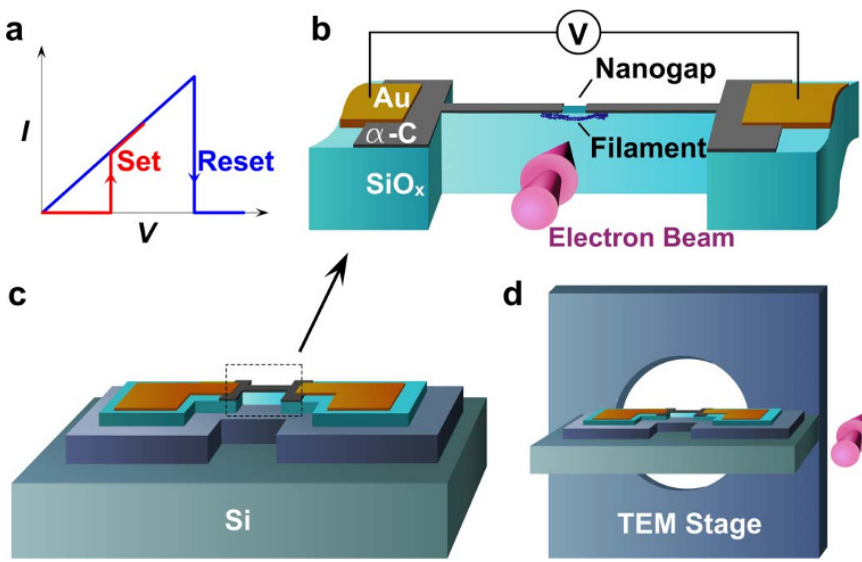

Figure $1 \mid I$ - $V$ behaviors and general device embodiments. (a) Schematic of the unipolar switching in $\mathrm{SiO}_{\mathrm{x}}$, showing the typical set (red) and reset (blue) $I$ - $V$ curves (9). (b) Schematic of the $\mathrm{SiO}_{\mathrm{x}}$ thin-walled structure for in situ TEM imaging. The switching region in $\mathrm{SiO}_{\mathrm{x}}$ is localized by the nanogap generated in the $\alpha$-C layer and imaged by TEM. (c) and (d) Schematics of the multi-stage structure and its arrangement with respect to the TEM stage. The pink arrows indicate the TEM electron beam imaging direction.

external electrical inputs (Fig. 1b). By electrical breakdown in the $\alpha-C$ layer, a disruption region or nanogap can be produced as we described previously in planar carbon-coated $\mathrm{SiO}_{\mathrm{x}}$ devices ${ }^{12}$. The broken ends of $\alpha$-C layer then serve as the electrodes for the $\mathrm{SiO}_{\mathrm{x}}$ in the nanogap region. The use of $\alpha-\mathrm{C}$ as the electrode material eliminates possible extrinsic effects from metals ${ }^{11}$, and the electrical breakdown-generated nanogap provides an easy method for the fabrication of closely spaced electrodes atop a thin-walled structure. The confinement from the nanogap pre-localizes the switching site so that it can be constantly monitored from before the electroforming or filament formation and throughout the experiment. During imaging, the electron beam from the TEM system travels perpendicularly across the $\mathrm{SiO}_{\mathrm{x}}$ thin-walled structure. In this configuration, the $\mathrm{SiO}_{\mathrm{x}}$ and the $\alpha-\mathrm{C}$ layer are spatially separated in the imaging plane, minimizing possible interference from the electrode material. Practically, for the successful passage of the electron beam across the nanogap region, a multi-stage design with the $\mathrm{SiO}_{\mathrm{x}}$ thin-walled structure is required (Fig. 1c). This multi-stage device is then vertically mounted on a home-built TEM stage (Supplementary Figs. S1, S2) that is capable of in situ electrical characterization (Fig. 1d). Note that while high vacuum environment ( $\sim 10^{-8}$ Torr) is involved in the TEM system, the multi-stage devices also work in the regular vacuum environment $\left(\sim 10^{-5}\right.$ Torr $)$ in a probe station as was adopted for typical device testing ${ }^{9,12}$. It shows the same resistive switching behaviors (Supplementary Fig. S3) with no $\mathrm{SiO}_{\mathrm{x}}$ thin-wall thickness dependence, indicating the same switching mechanism intrinsic to $\mathrm{SiO}_{\mathrm{x}}$ as reported previously.

Figs. 2a-d shows a series of scanning electron microscopy (SEM) images of the multi-stage device (see Supplementary Fig. S2 for fabrication details). The thickness of the $\mathrm{SiO}_{\mathrm{x}}$ thin-walled structure is $\sim 100 \mathrm{~nm}$ for the electron-beam transparency, and the length is $\leq 1 \mu \mathrm{m}$ to reduce the resistance of the $\alpha$-C electrodes $(\sim 20 \mathrm{~nm}$ thick). The successful imaging of the pristine structure by TEM is shown in Fig. 2e. After electrical breakdown in the $\alpha$-C layer, a nanogap is generated in the $\alpha$-C layer atop the $\mathrm{SiO}_{\mathrm{x}}$ thin-walled structure (Fig. 2f).

Fig. 3 shows a series of high-resolution TEM images of the nanogap region (right panels) with respect to different $I-V$ responses (left panels). Note that during the electrical characterization, the electron beam was temporarily blocked to exclude beam impact ${ }^{13}$. Immediately after the electrical breakdown in the $\alpha$-C layer, a nanogap of $\sim 15 \mathrm{~nm}$ is formed (Fig. 3a). Both the $\mathrm{SiO}_{\mathrm{x}}$ at the nanogap
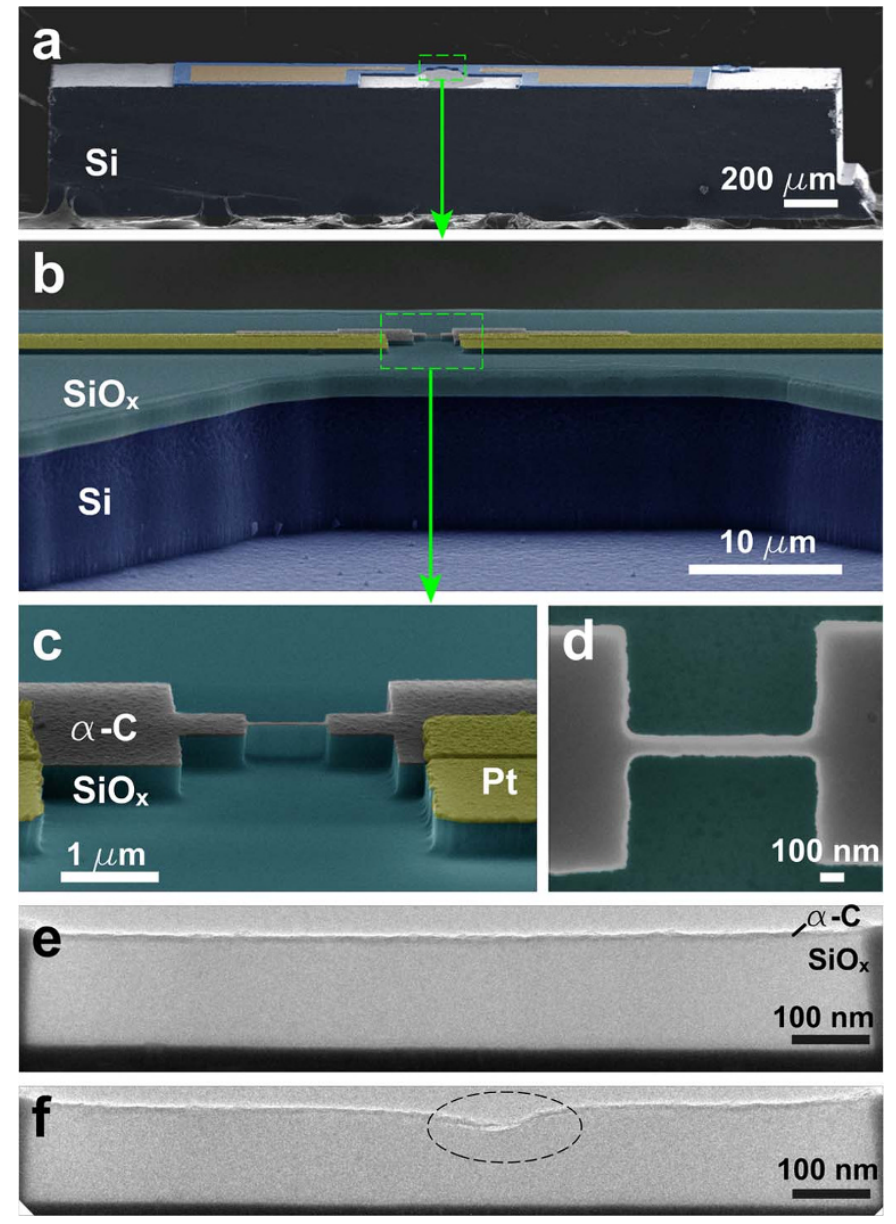

Figure $2 \mid$ SEM false-color images and TEM images of the electronic device. (a) -(c) Perspective SEM images of the multi-stage device for in situ imaging. (d) A top view of the $\mathrm{SiO}_{\mathrm{x}}$ thin-walled structure. (e) Low-magnification TEM image of the $\mathrm{SiO}_{\mathrm{x}}$ thin-walled structure with the $\alpha$-C layer on top. (f) The same structure as in (e) with the $\alpha$-C layer subjected to electrical breakdown. The circled region shows the nanogap in the $\alpha$-C layer.

region and the $\mathrm{SiO}_{\mathrm{x}}$ far from the nanogap show amorphous silica features. Because of the disruption in the $\alpha$-C layer, the device shows little conduction during the subsequent voltage sweep, until at $\sim 12 \mathrm{~V}$ the current suddenly increases (light grey curve in the plot of Fig. 3b). This conductance increase features the beginning of the electroforming process in $\mathrm{SiO}_{\mathrm{x}}{ }^{9}$. The device is subsequently electroformed, showing the characteristic $I-V$ curve featuring current increase (at $\sim 5 \mathrm{~V}$ ) and decrease (at $\sim 10 \mathrm{~V}$ ) that define the typical set and reset processes, respectively (grey curve). The device is set to the ON state (red curve). The immediate TEM imaging shows morphological changes at the nanogap region (Fig. 3b), as is often associated with the electroforming process ${ }^{8,9,14}$. Specifically, out of the amorphous background, $\sim 3 \mathrm{~nm}$ regions of nanocrystalline structure (based on the appearance of lattice fringes) appear at the nanogap (bounded region and inset in Fig. 3b). The apparent lattice spacing of the nanocrystal based on the fringes is distinct from that of the $\alpha-C$, indicating a different material form.

Due to the beam impact (see below), the formed ON and switching states degrade after imaging (light grey curve in Fig. 3c). A subsequent re-electroforming process is involved to set the device back to ON (grey and red curves). The immediate imaging shows growth in the nanocrystal (bounded region and inset in Fig. 3c). The partially degraded ON state after this imaging is compensated by a set process (red curve in Fig. 3d). For the subsequent voltage sweep to $14 \mathrm{~V}$, a sudden decrease in the conductance occurs at $\sim 12 \mathrm{~V}$, featuring the 

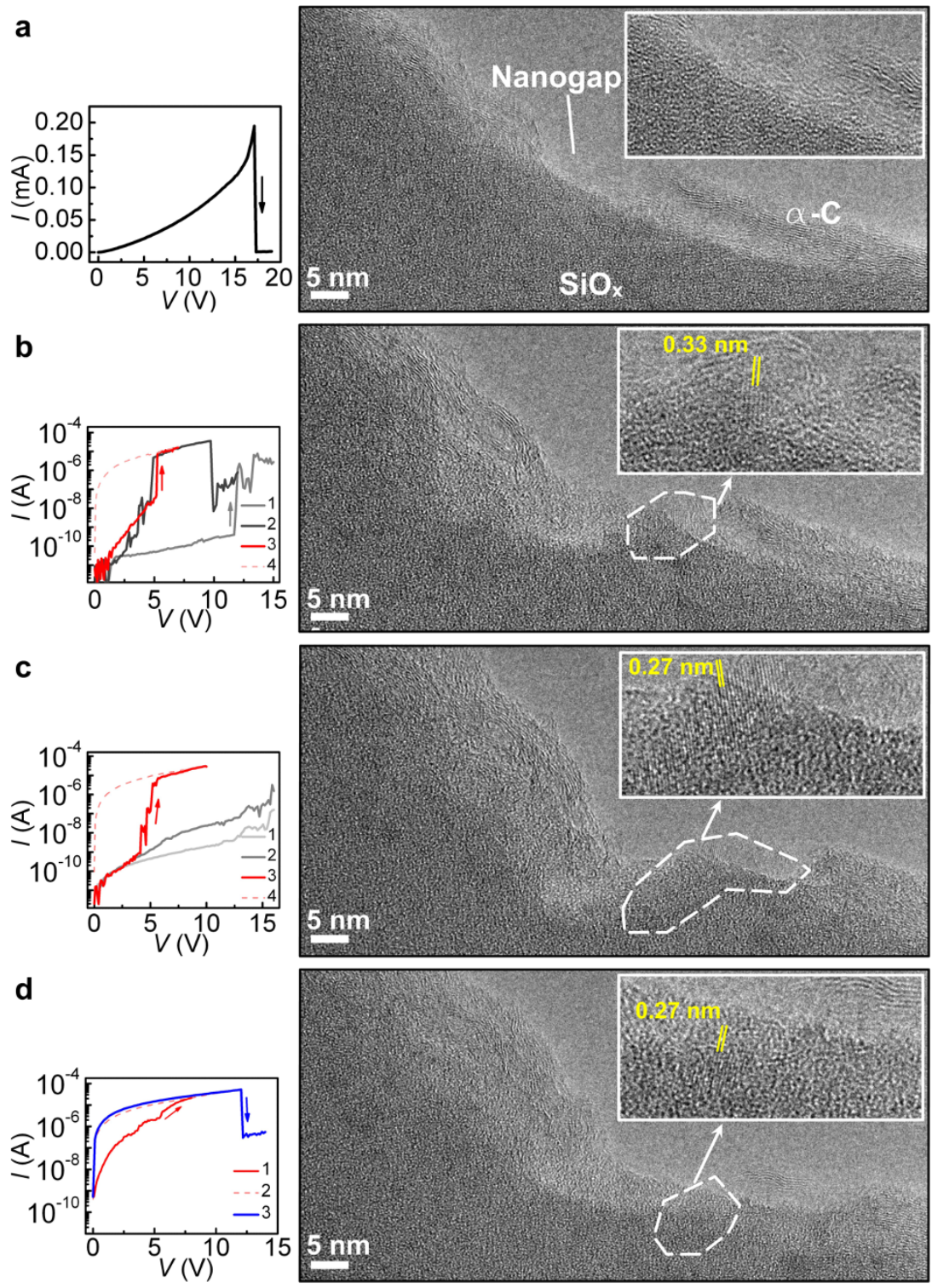

Figure $3 \mid$ High-resolution TEM images of the nanogap region (right panels) taken immediately after the electrical characterizations (left panels). (a) The electrical breakdown $I$ - $V$ curve in the $\alpha$-C layer and the formed nanogap. The inset shows the enlarged nanogap region which shows only amorphous features. (b) The electroforming process in $\mathrm{SiO}_{\mathrm{x}}$ with the state set to ON. The inset is an enlarged image of the circled region, showing nanocrystalline features. (c) A re-electroforming process in $\mathrm{SiO}_{\mathrm{x}}$ after the imaging in (b), with the state set to ON. The inset shows the enlarged image of the circled region, showing the growth of the nanocrystal. (d) A reset process after the imaging performed in (c). The inset shows the enlarged image of the circled region, showing the shrinkage of size in the nanocrystal. The numbers in all the left-panel plots indicate the voltage-sweep orders.

typical reset process 9 . The immediate imaging shows prominent shrinkage in the size of the nanocrystal (bounded region and inset in Fig. 3d).

\section{Discussion}

The nanocrystalline structures were persistently observed only at the nanogap region in the switching devices, indicating their correlation with the electroforming and switching processes. Selected area electron diffraction shows that the crystalline structures are consistent with $\mathrm{Si}$ nanocrystals (NCs) and not silicon carbide (Supplementary Fig. S4). As $\mathrm{SiO}_{\mathrm{x}}$ is the only source containing the $\mathrm{Si}$ element, the formation of the $\mathrm{Si}$ NCs shows that the energetically viable $\mathrm{SiO}_{\mathrm{x}} \rightarrow \mathrm{Si}$ process is associated with the electroforming process. The in situ recording of this process also excludes the possibility of processing-induced formation during the filament isolation as was involved in ex situ imaging. This TEM imaging process was studied over four different devices (Supplementary Figs. S4, S5), all yielding similar information.
As the ON state shows largely metallic conduction ${ }^{15}$, questions arise regarding the composition of the conductor since conventional silicon is semiconducting. Lattice-spacing measurements and electron diffraction patterns from the $\mathrm{Si}$ NCs show evidence of structural deviation from the conventional diamond cubic Si-I phase. As shown in Fig. 4, the intersected lattice spacing of 0.33 and $0.32 \mathrm{~nm}$ with a nearly perpendicular angle is the feature of the Si-III phase ${ }^{16}$, which is semi-metallic ${ }^{17}$ and often associated with other conducting phases ${ }^{18}$. This indication can also be seen in Fig. 3; while the lattice spacing of $0.27 \mathrm{~nm}$ can be assigned to Si-I(200), Si-III(211) or Si-XII(11-2) faces, that of $0.33 \mathrm{~nm}$ (Fig. 3b) can only be associated with Si-III/ $\mathrm{Si}$-XII phases ${ }^{16}$. In particular, as $\mathrm{Si}-\mathrm{I}(200)$ is the forbidden plane for diffraction ${ }^{19}$ and multiple scattering is considerably weakened at the nanoscale crystalline size, the strong signal of $0.27 \mathrm{~nm}^{-1}$ in the diffraction patterns is further indication of the conducting $\mathrm{Si}$-III/Si-XII phases $^{20}$ (Supplementary Figs. S4, S5). While these phase transitions in $\mathrm{Si}$ are typically induced by mechanical pressurization ${ }^{18}$, here it is 


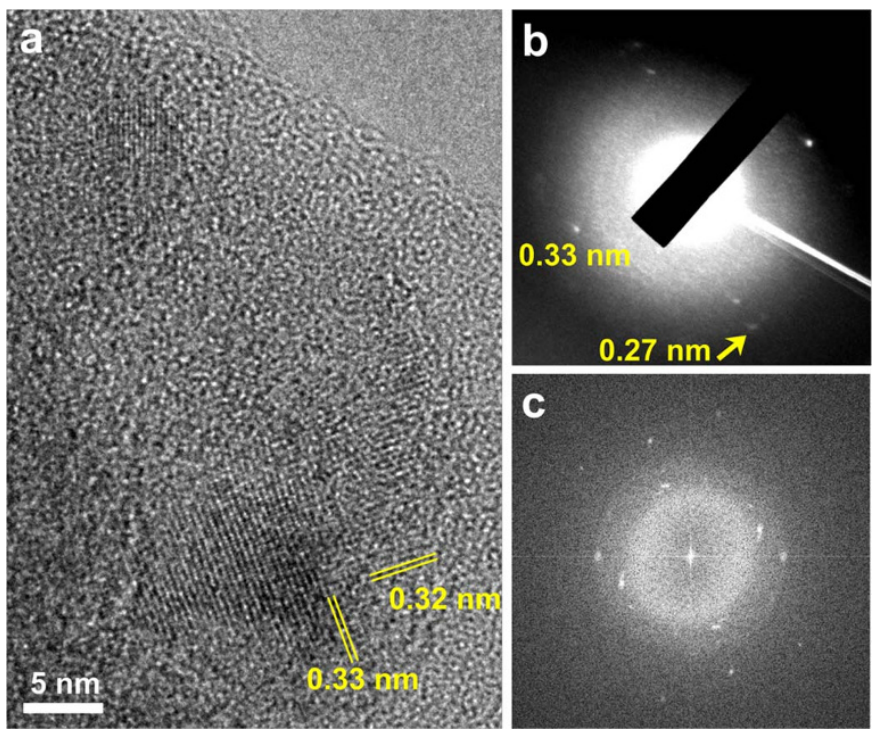

Figure 4| TEM and diffraction patterns from a different device. (a) High-resolution TEM image of the nanogap region from another $\mathrm{SiO}_{\mathrm{x}}$ switching device. (b) Selected-area diffraction pattern of the region shown in (a). (c) A fast Fourier transformed micrograph of the image shown in (a).

possible that the high electric field attained at the nanogap is inducing these phases. Indeed, the pressure induced by high electric field was revealed to induce structural distortion in silicon crystals ${ }^{21}$. The stability of the formed silicon phases at ambient environment ${ }^{17,18}$ also accounts for the electronically nonvolatile property in $\mathrm{SiO}_{\mathrm{x}}$ resistive switching memory. Remarkably, the suppression of the electroforming and switching in $\mathrm{SiO}_{\mathrm{x}}$ at low temperature ${ }^{9,15}$ coincides with the fact that the Si-III phase cannot be formed at liquid-nitrogen temperature $^{22}$. Furthermore, the semi-metallic NCs suggest a rationale for failure to induce gating in three-terminal embodiments of these devices $^{23}$, which was originally proposed to be carbon switching ${ }^{23}$ and later clarified as $\mathrm{SiO}_{\mathrm{x}}$-derived switching ${ }^{9,12}$. Note that while the elementary composition of the Si filament was identified in our previous ex situ imaging study ${ }^{9}$, its structural variation from the conventional Si form was not revealed due to the absence of a diffraction pattern. This revelation could have been further hindered by the alteration of the Si forms during sample preparation by FIB in the ex situ imaging, as the ion beam can potentially induce phase transitions in the Si forms ${ }^{16}$.

From the devices studied, the filament in $\mathrm{SiO}_{\mathrm{x}}$ is not in the form of a continuous single crystal across the nanogap, as seen in $\mathrm{TiO}_{\mathrm{x}}$ switching systems ${ }^{8}$. Instead, discrete $\mathrm{Si}$ NCs form across the nanogap. Imaging such a filament is challenging, because of its 3-dimensional structure (e.g., having different depth profiles perpendicular to the imaging plane), different crystalline orientations, and the possibility of narrower filamentary constrictions (e.g. $\leq 1 \mathrm{~nm}$ ) that are difficult to resolve from the environment. The growth of the Si NCs (Fig. 3c) indicates the general Si-rich nature along the nanogap in the electroformed device. This Si enrichment is also evidently correlated with the indention and shrinkage of the volume at the nanogap region, likely a result of oxygen outgassing ${ }^{14}$. The shrinkage of the Si NCs with respect to the conductance drop (Fig. 3d) indicates the possible amorphization process. This is consistent with the thermally induced amorphization observed in the metallic Si phases ${ }^{18,20}$, as the reset process in unipolar resistive switching is largely thermally stressdriven $^{2,9,24}$. In particular, resistance increase is associated with the amorphization process ${ }^{18}$. This provides a possible scenario for the filament rupture in the reset process.

We further take the electron-beam impact into consideration during the mechanistic interpretations. As mentioned above, the electron beam from the TEM system tends to degrade the conduction and switching state in $\mathrm{SiO}_{\mathrm{x}}$. This does not indicate a charge-based mechanism though, as that was ruled out in the x-ray irradiation experiment and high temperature stability measurements ${ }^{9}$. In fact, knockon structural change in $\mathrm{Si}$ can be readily induced by an electron beam at the imaging energy $(200 \mathrm{KeV})^{25}$, and the amorphization process can be induced ${ }^{16,26}$. This accounts for the switching degradation after beam exposure (Fig. 3b) as structural changes along the entire filament are induced. While this is a further indication of structural change-induced conductance switching in the Si filament, it also implies that the structural transition needed for the switching can be subtle.

As was mentioned before, the $\mathrm{SiO}_{\mathrm{x}}$ resistive switching system shows a larger reset voltage than the set value (i.e., $\mathrm{V}_{\text {reset }}>$ $\left.\mathrm{V}_{\text {set }}\right)^{9,12,27}$, which is different from typical unipolar systems that have $\mathrm{V}_{\text {set }}>\mathrm{V}_{\text {reset }}{ }^{1,2,28}$. We suggest that the electric-field effect and thermal effect (by current local heating) are largely responsible for the set and reset processes, respectively, and that the competing process between the two effects results in this different behavior. The $\mathrm{SiO}_{\mathrm{x}}$ resistive switching system features a lower $\mathrm{ON}$-state conductance compared to other unipolar systems ${ }^{28}$. As a result, no current compliance is needed in the set process to suppress the thermal reset effect. The thermally driven reset process is induced at a higher voltage $V_{\text {reset }}$ $\left(\mathrm{V}_{\text {reset }}>\mathrm{V}_{\text {set }}\right)$. Note that competition between the two processes persists in the region $\mathrm{V}>\mathrm{V}_{\text {reset }}$, with the thermal reset effect constantly overriding the set process driven by the field effect. This dynamic competition is evident from the large current fluctuations in the $\mathrm{V}>\mathrm{V}_{\text {reset }}$ region, which sometimes even shows transient $\mathrm{ON}$ state fluctuations (Supplementary Fig. S3a). This competition also explains why during a backward sweep the system goes back into $\mathrm{ON}$ state below $\mathrm{V}<\mathrm{V}_{\text {reset }}$ (ref. 9,12,27), because the field is sufficient to set the system to $\mathrm{ON}$ at $\mathrm{V}>\mathrm{V}_{\text {set }}$ whereas the thermal effect is too weak to prevent this at $\mathrm{V}<\mathrm{V}_{\text {reset. }}$. Note that while the thermal effect has been generally deemed responsible for the reset process in unipolar resistive switching systems $s^{1,30}$, no direct observation has been made ${ }^{30}$. Our in situ study provides further evidence and insights for this process.

In summary, the study here provides an overall picture of the intrinsic resistive switching in $\mathrm{SiO}_{\mathrm{x}}$. The electroforming is through the $\mathrm{SiO}_{\mathrm{x}} \rightarrow \mathrm{Si}$ process with the semi-metallic Si state identified. The switching is indicated to be through the transition between the semimetallic and amorphous Si forms. It also provides a general overview of electrical breakdown in silicon oxides. It should be noted that while a redox process was proposed to be responsible for the switching in our previous study ${ }^{9}$, the current study suggests switching based on structural transitions of the Si. The degradation of the resistive switching state to a nonswitchable metallic state (hard breakdown ${ }^{29}$ ) in $\mathrm{SiO}_{\mathrm{x}}$ is likely to be associated with the further aggregation of the metallic Si forms. The method described here can also be applied to other resistive switching materials for mechanistic investigation.

\section{Methods}

The multi-stage $\mathrm{SiO}_{\mathrm{x}}$ thin-wall structure as shown in Fig. 2 is fabricated from a silicon wafer (thickness $\sim 500 \mu \mathrm{m}$ ) capped with $2 \mu \mathrm{m}$ thermal $\mathrm{SiO}_{\mathrm{x}}(x \sim 2)$ on top. The $\alpha-\mathrm{C}$ layer was grown by chemical vapor deposition method at $900^{\circ} \mathrm{C}$ using $\mathrm{C}_{2} \mathrm{H}_{2}$ as precursor gas. Electron-beam lithography, photolithography and reactive ion etching steps were involved for the definition of the multi-stage $\mathrm{SiO}_{\mathrm{x}}$ imaging structure (see Supplementary Information for more details). The fabricated structure was then mounted to a home-built TEM stage (Supplementary Figure S1) that is capable of electrical input, and electrically connected (through wire bonding) to an Agilent B1500 semiconductor parameter analyzer for the electrical characterizations. The imaging is carried out on a JEM-2100F TEM system with the beam energy at $200 \mathrm{KeV}$

1. Waser, R. \& Aono, M. Nanoionics-based resistive switching memories. Nat. Mater. 6, 833-840 (2007)

2. Sawa, A. Resistive switching in transition metal oxides. Materials Today 11, 28-36 (2008). 
3. Lee, M.-J. et al. A fast, high-endurance and scalable non-volatile memory device made from asymmetric $\mathrm{Ta}_{2} \mathrm{O}_{5-\mathrm{x}} / \mathrm{TaO}_{2-\mathrm{x}}$ bilayer structures. Nat. Mater. 10, 625630 (2011).

4. Meijer, G. I. Who wins the nonvolatile memory race? Science 319, 1625-1626 (2008).

5. Choi, B. J. et al. Resistive switching mechanism of $\mathrm{TiO}_{2}$ thin films grown by atomic-layer deposition. J. Appl. Phys. 98, 033715 (2005).

6. Szot, K., Speier, W., Bihlmayer, G. \& Waser, R. Switching the electrical resistance of individual dislocations in single-crystalline $\mathrm{SiTiO}_{3}$. Nat. Mater. 5, 312-320 (2006).

7. Liu, C. N., Stewart, D. R., Williams, R. S. \& Bockrath, M. Direct observation of nanoscale centers in metal/molecule/metal structures. Nano Lett. 4, 569-572 (2004).

8. Kwon, D. H. et al. Atomic structure of conducting nanofilaments in $\mathrm{TiO}_{2}$ resistive switching memory. Nat. Nanoetchnol. 5, 148-153 (2010).

9. Yao, J., Sun, Z., Zhong, L., Natelson, D. \& Tour, J. M. Resistive switches and memories from silicon oxide. Nano Lett. 10, 4105-4110 (2010).

10. Yang, Y. C., Pan, F., Liu, Q., Liu, M. \& Zeng, F. Fully room-temperature-fabricated nonvolatile resistive memory for ultrafast and high-density memory application. Nano Lett. 9, 1636-1643 (2009).

11. Schindler, C., Thermadam, S. C., Waser, R. \& Kozicki, M. N. Bipolar and unipolar resistive switching in $\mathrm{Cu}$-doped $\mathrm{SiO}_{2}$. IEEE Trans. Electron Devices 54, 2762-2768 (2007)

12. Yao, J. et al. Resistive switching in nanogap systems on $\mathrm{SiO}_{2}$ substrates. Small $\mathbf{5}$, 2910-2915 (2009).

13. Du, X.-W., Takeguchi, M., Tanaka, M. \& Furuya, K. Formation of crystalline Si nanodots in $\mathrm{SiO}_{2}$ films by electron irradiation. Appl. Phys. Lett. 82, 1108 (2003).

14. Yang, J. J. et al. The mechanism of electroforming of metal oxide memristive switches. Nanotechnol. 20, 215201 (2009).

15. Dearnaley, G., Stoneham, A. M. \& Morgan, D. V. Electrical phenomena in amorphous oxide films. Rep. Prog. Phys. 33, 1129 (1970).

16. Ge, D., Dominich, V. \& Gogotsi, Y. High-resolution transmission electron microscopy study of metastable silicon phases produced by nanoindentation. J. Appl. Phys. 93, 2418 (2003).

17. Besson, J. M., Mokhtari, E. H., Gonzalez, J. \& Weill, G. Electrical properties of semimetallic silicon III and semiconductive silicon IV at ambient pressure. Phys. Rev. Lett. 59, 473 (1987).

18. Ruffell, S., Sears, K., Bradby, J. E. \& Williams, J. S. Room temperature writing of electrically conductive and insulating zones in silicon by nanoindentation. Appl. Phys. Lett. 98, 052105 (2011).

19. Colella, R. \& Merlini, A. A study of the (222) "forbidden" reflection in germanium and silicon. Phys. Stat. Sol. 18, 157 (1966).

20. Ge, D., Domnich, V. \& Gogotsi, Y. Thermal stability of metastable silicon phases produced by nanoindentation. J. Appl. Phys. 95, 2725 (2004).

21. Aboyan, A. O. \& Aghbalyan, S. G. Structural distortion of semiconducting silicon crystals caused by constant electric field. Cryst. Res. Technol. 45, 140-144 (2010).

22. Khayyat, M. M., Banini, G. K., Hasko, D. G. \& Chaudhri, M. M. Raman microscopy investigations of the structural phases transformation in crystalline and amorphous silicon due to indentation with a Vickers diamond at room temperature and at 77 K. J. Phys. D: Appl. Phys. 36, 1300 (2003)
23. Li, Y., Sinitskii, A. \& Tour, J. M. Electronic two-terminal bistable graphitic memories. Nat. Mater. 7, 966-971 (2008)

24. J. C. Phillips. Internal stresses and formation of switchable nanowires at thin silica film edges. J. Appl. Phys. 109, 034312 (2011).

25. Du, X.-W., Wang, B., Zhao, N. Q. \& Furuya, F. Structure evolution of silicon nanocrystals under electron irradiation. Scripta Materialia 53, 899-903 (2005).

26. Takeda, S. \& Yamasaki, J. Amorphization in silicon by electron irradiation. Phys Rev. Lett. 83, 320 (1999).

27. Yao, J., Zhong, L., Natelson, D. \& Tour, J. M. Silicon oxide: a non-innocent surface for molecular electronics and nanoelectronics studies. J. Am. Chem. Soc. 133, $941-$ 948 (2011).

28. Kim, D. C. et al. Electrical observations of filamentary conductions for the resistive memory switching in NiO films. Appl. Phys. Lett. 88, 202102 (2006).

29. Dimaria, J. D., Cartier, E. \& Arnold, D. Impact ionization, trap creation, degradation, and breakdown in silicon dioxide films on silicon. J. Appl. Phys. 73, 3367 (1993).

30. Kim, K. M., Jeong, D. S. \& Hwang, C. S. Nanofilamentary resistive switching in binary oxide system; a review on the present status and outlook. Nanotechnology 22, 254002 (2011).

\section{Acknowledgements}

D. N. acknowledges the support of the David and Lucille Packard Foundation. L. Z acknowledges support from the Texas Instruments Leadership University Fund and National Science Foundation Award No. 0720825. J. M. T. acknowledges support from the Army Research Office (\# W911NF-08-C-0133, any opinions, findings and conclusions or recommendations expressed in this material are those of the author(s) and do not necessarily reflect the views of the U.S. Army Research Office) through the SBIR program administrated by PrivaTran, LLC. C. Kittrell aided in the design of the TEM probe device.

\section{Author contributions}

J. Y. designed and carried out the experiment, did the data analysis and wrote the paper. L. Z., D. N. and J. M. T. oversaw the project and edited the manuscript.

\section{Additional information}

Supplementary information accompanies this paper at http://www.nature.com/ scientificreports

Competing financial interests: The authors declare no competing financial interests.

License: This work is licensed under a Creative Common Attribution-NonCommercial-ShareAlike 3.0 Unported License. To view a copy of this license, visit http://creativecommons.org/licenses/by-nc-sa/3.0/

How to cite this article: Yao, J., Zhong, L., Natelson, D. \& Tour, J.M. In situ imaging of the conducting filament in a silicon oxide resistive switch. Sci. Rep. 2, 242; DOI:10.1038/ srep00242 (2012). 